\title{
Protocolo clínico e diretrizes terapêuticas em doença de Chagas: perspectivas e desafios para o Brasil
}

O Brasil deu um passo muito importante em 2018 para a ampliação do acesso a diagnóstico e a tratamento das pessoas acometidas pela doença de Chagas com a publicação do Protocolo Clínico e Diretrizes Terapêuticas (PCDT) em doença de Chagas. As estimativas mais atuais, referendadas pelo $2^{\circ}$ Consenso Brasileiro em doença de Chagas, revelam o tamanho deste desafio: 1,9 milhão a 4,6 milhões de pessoas infectadas por Trypanosoma cruzi ${ }^{1}$ que poderiam ser beneficiadas no Brasil. De fato, a doença de Chagas representa a doença tropical negligenciada com a maior carga de morbimortalidade no país, ${ }^{2,3}$ o que torna este documento de elevado impacto, como referência internacional para outros países endêmicos e não endêmicos.

O seu processo de construção foi iniciado em 2016, tendo sido finalizado com a publicação da Portaria ${ }^{\circ} 57$ (SCTIE/MS n ${ }^{\circ} 57 / 2018$ ) no Diário Oficial da União (DOU) número 210 de 31 de outubro de 2018, ${ }^{4}$ que registrou a decisão de aprová-lo no âmbito do Sistema Único de Saúde (SUS). Assinada pela Secretaria de Ciência, Tecnologia e Insumos Estratégicos do Ministério da Saúde, esta portaria deu publicidade ao relatório de recomendação da Comissão Nacional de Incorporação de Tecnologias no SUS (CONITEC). ${ }^{5}$

A CONITEC é um órgão colegiado de caráter permanente, integrante da estrutura regimental do Ministério da Saúde, que tem por objetivo assessorar o Ministério da Saúde nas atribuições relativas à incorporação, exclusão ou alteração pelo SUS de tecnologias em saúde, bem como na constituição ou alteração do PCDT. ${ }^{6}$ Os PCDTs são documentos que estabelecem critérios para “diagnóstico de doenças ou agravos à saúde; tratamentos preconizados, com os medicamentos e demais produtos apropriados, quando couber; posologias recomendadas; mecanismos de controle clínico; e acompanhamento e verificação dos resultados terapêuticos, a serem seguidos pelos gestores do SUS". ${ }^{7}$ Como referência nacional, este documento deve ser baseado em evidência científica criteriosa e deve considerar critérios de eficácia, segurança, efetividade e custo-efetividade das tecnologias recomendados. Trata-se de uma ferramenta estratégica para o SUS pois propicia mecanismos para o monitoramento clínico quanto à efetividade do tratamento e à ocorrência de efeitos adversos, além de auxiliar como instrumento de apoio na disponibilização de medicamentos e procedimentos. A atualização dos PCDTs é prevista a cada dois anos, devendo ser oportunidade para revisão à luz dos avanços técnico-científicos e políticos sobre o tema no país. ${ }^{6,7}$

Após a identificação e priorização de tema, com definição do escopo ${ }^{8}$ realizou-se em abril de 2016 enquete pública sobre a primeira etapa de proposta de elaboração deste PCDT destinado à atenção e ao cuidado da pessoa com doença de Chagas no Brasil. A finalidade da enquete foi possibilitar a participação da sociedade desde a primeira etapa da elaboração do PCDT de definição de escopo. ${ }^{8}$ Ao longo do período de 2016 a 2018 várias reuniões foram realizadas até que se alcançasse sua aprovação final, após nova consulta pública em 2018.

O documento traz avanços de alta relevância para ampliar o reconhecimento das pessoas afetadas pela doença de Chagas na rede de atenção do SUS, ampliando o acesso não apenas para diagnóstico, mas também para o tratamento da doença (tanto em termos parasitários quanto das complicações clínicas) em busca de uma atenção integral. ${ }^{5}$

Trata-se de um documento histórico de expressivo avanço na integração do tema nas políticas públicas dentro do SUS. Possibilita ainda avanços rumo ao alcance do direito à saúde das pessoas com doença de Chagas e suas famílias. ${ }^{5,9}$ Entretanto, apesar da conquista, ainda há muito o que se fazer para a implementação no contexto real da rede de atenção à saúde das recomendações do PCDT, salvaguardando-se, portanto, as garantias para o seu alcance de fato..$^{9,10}$

Ressalta-se a recomendação neste documento da implementação do diagnóstico sorológico da infecção por $T$. cruzi no país por meio de estratégias adequadas de rastreamento dentro de contextos epidemiológicos de maior risco e sem limitação etária, incluindo o âmbito do pré-natal. ${ }^{5,11}$ Além disto, o PCDT reforçou a necessidade de integração do tratamento parasitológico para os casos indicados na rede de atenção do SUS, em particular na rede de atenção básica..$^{5}$ Ademais, as questões relativas à atenção integral perpassam, necessariamente, pelo adequado manejo das formas crônicas da doença na rede do SUS.,5,9 Daí a importância de os testes sorológicos serem oferecidos de forma ampla e integrada em toda a rede do SUS, em particular na rede de atenção básica, permitindo que o tratamento parasitológico e/ou sintomático, quando apropriadamente indicados, sejam capazes de reduzir a elevada carga de morbimortalidade associada às formas crônicas cardíaca e digestiva da doença. ${ }^{1-3,5,9}$

No PCDT em doença de Chagas é contundente a recomendação para a inclusão da doença de Chagas em sua fase crônica como um evento de notificação compulsória no Brasil. ${ }^{5}$ Justifica-se esta recomendação considerando-se sua relevância em saúde pública diante da magnitude expressa na carga de morbimortalidade, ${ }^{1-3,5,9}$ assim como de seu potencial de disseminação, sua transcendência, sua vulnerabilidade e dos compromissos internacionais assumidos, em particular aqueles contidos na agenda para 2030 dos Objetivos de Desenvolvimento Sustentável (ODS) em seu objetivo 3, meta 3.3. ${ }^{12}$ Esta recomendação é consenso entre os especialistas, mas aguarda ainda a sua implementação pelo Ministério da Saúde. Com a publicação do PCDT para doença de Chagas, espera-se que esta decisão seja tomada o mais brevemente possível. ${ }^{5,9,13}$ 


\section{REFERÊNCIAS}

1. Dias JC, Ramos AN Jr, Gontijo ED, Luquetti A, Shikanai-Yasuda MA, Coura JR, et al. 2nd Brazilian Consensus on Chagas Disease. Rev Soc Bras Med Trop. 2016;49(Suppl.1):3-60.

2. Martins-Melo FR, Carneiro M, Ramos AN Jr, Heukelbach J, Ribeiro AL, Werneck GL. The burden of Neglected Tropical Diseases in Brazil, 1990-2016: a subnational analysis from the Global Burden of Disease Study 2016. PLoS Negl Trop Dis. 2018;12(6):e0006559.

3. Brasil. Ministério da Saúde. Secretaria de Vigilância em Saúde. Departamento de Vigilância de Doenças e Agravos não Transmissíveis e Promoção da Saúde. Saúde Brasil 2017: uma análise da situação de saúde e os desafios para o alcance dos objetivos de desenvolvimento sustentável [Internet]. Brasília: Ministério da Saúde; 2018 [acesso em: 19 dez 2018]. Disponível em: http://bvsms.saude.gov.br/bvs/publicacoes/ saude_brasil_2017_analise_situacao_saude_desafios_objetivos_desenvolvimento_sustetantavel.pdf

4. Comissão Nacional de Incorporação de Tecnologias no SUS (CONITEC). Portaria n 57, de 30 de outubro de 2018. Torna pública a decisão de aprovar o Protocolo Clínico e Diretrizes Terapêuticas da doença de Chagas, no âmbito do Sistema Único de Saúde - SUS [Internet]. [Brasília]: CONITEC; 2018 [acesso em: 19 dez 2018]. Disponível em: http://conitec.gov.br/images/Relatorios/Portaria/2018/PortariasSCTIE_44-45_50a54_56a61.pdf

5. Comissão Nacional de Incorporação de Tecnologias no SUS (CONITEC). Relatório 397 - Protocolo Clínico e Diretrizes Terapêuticas da doença de Chagas [Internet]. Brasília: CONITEC; 2018 [acesso em: 19 dez 2018]. Disponível em: http://conitec.gov.br/images/Relatorios/2018/ Recomendacao/Relatorio_PCDT_Doenca_de_Chagas.pdf

6. Comissão Nacional de Incorporação de Tecnologias no SUS (CONITEC). A comissão [Internet]. Brasília: CONITEC; 2015 [acesso em 19 dez 2018]. Disponível em: http://conitec.gov.br/entenda-a-conitec-2

7. Comissão Nacional de Incorporação de Tecnologias no SUS (CONITEC). Protocolos e Diretrizes [Internet]. Brasília: CONITEC; 2016 [acesso em 19 dez 2018]. Disponível em: http://conitec.gov.br/index.php/protocolos-e-diretrizes

8. Comissão Nacional de Incorporação de Tecnologias no SUS (CONITEC). Comissão Nacional de Incorporação de Tecnologias no SUS. ESCOPO: Protocolo Clínico e Diretrizes Terapêuticas - Doença de Chagas [Internet]. Brasília: CONITEC; 2016 [acesso em 19 dez 2018 ]. Disponível em: http://conitec.gov.br/images/Relatorios/2016/PropostaEscopo_PCDT_DoencaChagas.pdf

9. Ramos AN Jr, Sousa AS. The continuous challenge of Chagas disease treatment: bridging evidence-based guidelines, access to healthcare, and human rights. Rev Soc Bras Med Trop. 2017;50(6):745-7.

10. Organización Panamericana de la Salud (OPS). Decálogo básico de la atención de Chagas a nivel primário [Internet]. Colonia del Sacramento: OPS; 2014 [acesso em $19 \mathrm{dez}$ 2018]. Disponível em: http://www.paho.org/hq/index.php?option=com_docman\&task=doc_download\&Itemid= \&gid=27245\&lang $=$ es

11. Organización Panamericana de la Salud (OPS). ETMI-PLUS: Marco para la eliminación de transmisión maternoinfantil del VIH, la sífilis, la hepatitis y la enfermedad de Chagas [Internet]. Colonia del Sacramento: OPS; 2017 [acesso em 19 dez 2018]. Disponível em: http://www.paho. org/hq/index.php?option=com_docman\&task $=$ doc_view\&gid=41278\&Itemid=270

12. Organização das Nações Unidas no Brasil (ONU). Documentos temáticos: objetivos de desenvolvimento sustentável 1, 2, 3, 5, 9 , 14 [Internet]. Brasília: ONU; 2017 [acesso em 19 dez 2018]. Disponível em: http://www.br.undp.org/content/brazil/pt/home/library/ods/documentos-tematicos-ods-1--2--3--5--9--14.html

13. Sociedade Brasileira de Medicina Tropical (SBMT). Protocolo clínico e diretrizes terapêuticas em Doença de Chagas: conquista trará avanços no controle da doença [Internet]. Brasília: SBMT; 2018 [acesso em 19 dez 2018]. Disponível em: http://www.sbmt.org.br/portal/clinicalprotocol-and-therapeutic-guidelines-on-chagas-disease-achievement-will-bring-advances-in-disease-control/

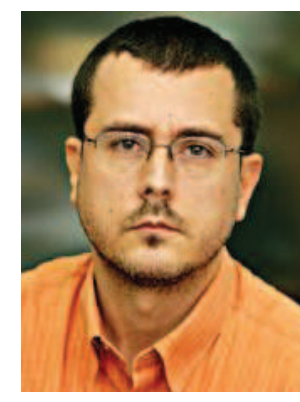

Dr Alberto Novaes Ramos Júnior Departamento de Saúde Comunitária Universidade Federal do Ceará

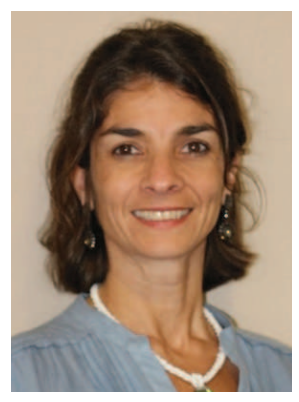

Dra Andréa Silvestre de Sousa Instituto Nacional de Infectologia Evandro Chagas Fundação Oswaldo Cruz

\section{Como citar:}

Ramos AN Jr, Sousa AS. Protocolo clínico e diretrizes terapêuticas em doença de Chagas: perspectivas e desafios para o Brasil. Rev Med UFC. 2018 out-dez;58(4):6-7. 